\title{
Measurement of the flavour composition of dijet events in pp collisions with ATLAS
}

\author{
Shima Shimizu* on behalf of the ATLAS collaboration \\ Kobe University \\ E-mail: shima.shimizu@cern.ch
}

\begin{abstract}
A measurement of the flavour composition of dijet events produced in $p p$ collisions at $7 \mathrm{TeV}$ using the ATLAS detector at the Large Hardron Collider (LHC) is presented. Six possible combinations of light, charm and bottom jets are identified in dijet events, where the jet flavour is defined by the presence of bottom, charm or solely light flavour hadrons in the jet. The results agree with the predictions of leading- and next-to-leading-order calculations, with the exception of the dijet fraction composed of bottom and light flavour jets, which is underestimated by all models at large jet transverse momentum. The difference between bottom jet production rates in leading and subleading jets is consistent with the next-to-leading-order predictions.
\end{abstract}

XXI International Workshop on Deep-Inelastic Scattering and Related Subject -DIS2013,

22-26 April 2013

Marseilles,France

\footnotetext{
* Speaker.
} 


\section{Introduction}

The production of jets containing bottom and charm hadrons has less influence by low energy hadronisation and is a topic of strong interest for understanding QCD. One can think of three mechanisms of heavy flavour production in a dijet system. The first one is heavy flavour quark pair creation, in which two heavy quarks are created in the hard interaction. This mechanism can be described by perturbative QCD. The second one is heavy flavour quark excitation. In this mechanism, a single heavy quark originated from a proton is in the hard interaction and therefore this is sensitive to the heavy-quark distribution in the proton. The last one is gluon splitting, where no heavy quark is in the hard interaction and a heavy quark pair is created from a gluon in the parton shower.

In this measurement, six flavour combinations in dijet events are considered, namely $B B, C C$, $U U, B U, C U$ and $B C$, where $B(C)$ is a bottom (charm) jet which is a jet including bottom (charm) hadrons and $U$ is a light jet including not only $u, d$ and $s$ quark jets but also gluon jets. The analysis aims to measure the fractions of the six combinations in dijet events, $f_{B B}, f_{C C}, f_{U U}, f_{B U}, f_{C U}$ and $f_{B C}$. Jet flavour is identified using templates which are derived from kinematics of secondary vertex in a jet. No explicit jet flavour tagging is done in this measurement.

This contribution describes the outline of the measurement performed using the ATLAS detector at the LHC. Further details of the measurement can be found at elsewhere [1].

\section{Analysis}

The measurement uses $39 \mathrm{pb}^{-1}$ of proton-proton collision data at a centre-of-mass energy $\sqrt{s}=7 \mathrm{TeV}$, collected using the ATLAS detector [2] in 2010. Events with a well reconstructed event vertex are selected. Jets in an event are reconstructed using the anti- $k_{t}$ algorithm with a jet size parameter of $R=0.4$. Jets with transverse momentum $p_{\mathrm{T}}>30 \mathrm{GeV}$ and rapidity $|y|<2.1$ are considered. Dijet events are selected by requiring an azimuthal angular separation between the two jets of $\Delta \phi>2.1 \mathrm{rad}$. In the following, the jet with the highest $p_{\mathrm{T}}$ in the event is referred to as the leading jet and the one with the second highest $p_{\mathrm{T}}$ is referred to as the subleading jet.

For the extraction of the flavour composition, a template fit is performed. The templates utilise kinematic information from secondary vertices in jets. The baseline templates are constructed using PYTHIA 6.423 dijet Monte Carlo (MC) [3], which utilises a leading-order (LO) $2 \rightarrow 2$ matrix element, with the MRST LO* [4] PDF set. The event generation uses the ATLAS Minimum Bias Tune 1 (AMBT1) [5] set of parameters. The generated events are passed through the full simulation [6] of the ATLAS detector based on GEANT4 [7].

A single secondary vertex is fitted to each jet and should have a positive decay length and total invariant mass larger than $0.4 \mathrm{GeV}$. Two-track vertices coming from $K_{S}^{0}$ and $\Lambda$ decays and from interactions in the detector material are removed. The reconstruction efficiencies of secondary vertices in leading and subleading jets are estimated using the MC simulation for light, charm and bottom jets. While the efficiencies in leading and subleading jets agree for charm jets and for bottom jets, the efficiency for light jets, which is actually the fake vertex reconstruction probability, is found to be higher for subleading jets. This difference is taken into account in the template fit described below. 
Two variables for the template fit are chosen to maximize sensitivity to the flavour content with minimal dependence on jet $p_{\mathrm{T}}$ and rapidity and on detector resolution. The variables are

$$
\Pi=\frac{m_{\mathrm{vertex}}-0.4 \mathrm{GeV}}{m_{B}} \cdot \frac{\sum_{\mathrm{vertex}} E_{i}}{\sum_{\mathrm{jet}} E_{i}}, \quad B=\frac{\sqrt{m_{B}} \cdot \sum_{\mathrm{vertex}}\left|\vec{p}_{\mathrm{T} i}\right|}{m_{\mathrm{vertex}} \cdot \sqrt{p_{\mathrm{T}} \mathrm{jet}}},
$$

where $i$ is for a charged particle, $m_{\mathrm{vertex}}$ is the invariant mass of the vertex and the $m_{B}=5.2794 \mathrm{GeV}$ constant is the average $B$-meson mass. The variable $\Pi$ is a product of mass and energy fraction of a secondary vertex and $B$ is a "boost" variable, which corresponds approximately to the relativistic $\gamma$ factor of a secondary vertex. They are transformed to the interval between 0 and 1 by

$$
\Pi^{T}=\frac{\Pi}{\Pi+0.04}, \quad B^{T}=\frac{B \cdot B}{B \cdot B+10 .} .
$$

These $\Pi^{T}$ and $B^{T}$ are found to be independent on jet rapidity and $\Pi^{T}$ has only weak jet $p_{\mathrm{T}}$ dependence. The boost variable $B^{T}$ is sensitive to the gluon splitting. Figure 1 shows the two-dimensional distributions of $\Pi^{T}$ and $B^{T}$. Clear separation of light, charm and bottom jets is seen.

A two-dimensional template is created based on these $\Pi^{T}$ and $B^{T}$. In addition to light-, charmand bottom-jet templates, a template for jets with two $b$-quarks inside is also created, since the prediction of the fraction of such jets may be incorrect in the PYTHIA MC. As the analysis utilises kinematic information of secondary vertices, its results strongly depend on the description of the resolution of track impact parameters. In order to have a better description of data, PYTHIA MC templates are tuned on data by mixing PYTHIA events with smeared track impact parameters.

A dijet analysis model is created with the following parameters. Each flavour composition in dijet events gives five free parameters, where $f_{U U}$ can be determined as the residual of the others. Scale factors are applied on the fake vertex reconstruction probability from the MC simulation for light jets and are considered as free parameters in the analysis. The scale factors are applied separately on the probabilities in the leading and subleading jet. The values of reconstruction efficiencies of a secondary vertex in bottom and charm jets are taken from the MC simulation. Presence of a heavy flavour quark in a jet can affect the jet properties and leads to asymmetry in production rates of dijet events with heavy flavour hadrons in the leading jets and in the subleading jets. This asymmetry in $B U$ dijet events is taken into account in the analysis with a free parameter $A_{b}=f_{B U}^{L} / f_{B U}^{S L}-1$, where $L(S L)$ indicates that the bottom jet is the leading (subleading) jet. Similar asymmetry parameter $A_{c}$ for charm jets in $C U$ dijet events is fixed to the MC prediction. The final
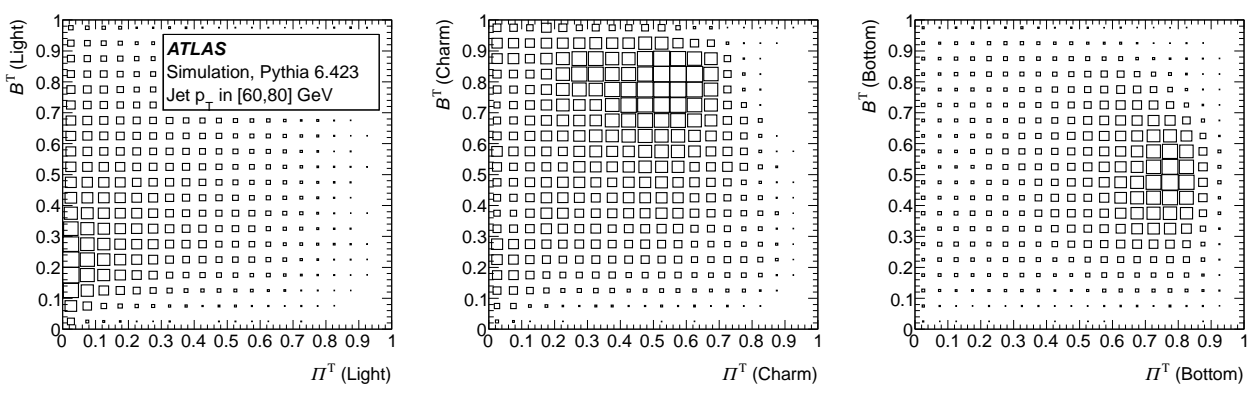

Figure 1: Two-dimensional $\Pi^{T}$ and $B^{T}$ distributions from the MC simulation for light, charm and bottom jets with $60<p_{\mathrm{T}}<80 \mathrm{GeV}$. [1] 
free parameter in the analysis is $b_{2}$ which is a parameter for relative mixture of the template for jets with two $b$-quarks inside compared to that for jets with a $b$-quark inside. No additional template for jets with two $c$-quarks inside is considered.

\section{Results}

Dijet events are analysed with the template fits with the nine free parameters described above. Both $\Pi^{T}$ and $B^{T}$ distributions in data are well reproduced by the fit results within $\pm 10 \%$, where the distributions are checked separately for dijet events with a single secondary vertex in leading jets, those with a single secondary vertex in subleading jets and those with two secondary vertices in both jets. Figure 2 shows the fit results of a few free parameters. Average fake vertex reconstruction probability in light jets in data is well reproduced by MC showing that the performance of the ATLAS detector is well understood in the MC simulation. The parameter $b_{2}$ is found to be significantly larger than zero indicating a large contribution of the additional template for jets with two $b$-quarks inside with respect to PYTHIA prediction. The contribution gets larger for the higher jet $p_{\mathrm{T}}$. The fitted value of the bottom jet asymmetry, $A_{b}$, is corrected to the particle-jet level and is compared to several MC predictions, where a particle-jet is a jet built from stable particles in MC event generation. The predictions are made by PYTHIA, HERWIG++ 2.4.2 [8], an LO MC, and POWHEG [9], a next-to-leading-order (NLO) matrix element MC interfaced to PYTHIA 6.423 for parton showering. The result shows that the POWHEG prediction gives the best description of the bottom jet asymmetry in data.

The results of the flavour composition are unfolded by bin-by-bin correction. Several sources of systematic uncertainties are considered, such as pileup effect, jet energy scale, secondary vertex reconstruction efficiencies, template shapes, imperfection of $\mathrm{MC}$ description in jet $p_{\mathrm{T}}$ and rapidity, influence of data-MC difference in tracking performance, influence from the fixed parameters for charm jets and unfolding. Uncertainties from the jet energy scale and template shapes mainly account for the total systematic uncertainties.

The measured flavour composition is shown in Figure 3. The fractions are generally well reproduced by both NLO and LO MC predictions, except for the $B U$ fraction. The measured $B U$ fraction is higher than any of the predictions at jet $p_{\mathrm{T}}>100 \mathrm{GeV}$.
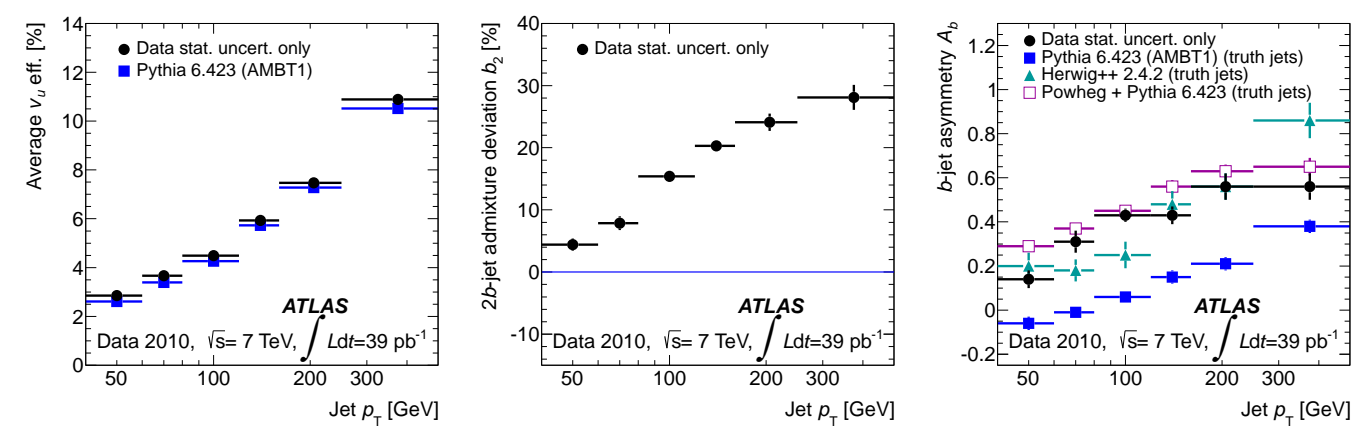

Figure 2: Fit results for the average fake vertex reconstruction probability in light jets, $v_{u}$, (left), the parameter $b_{2}$ for the mixture of the template for jets with two $b$-quarks inside (middle) and the bottom jet asymmetry $A_{b}$ (right). [1] 

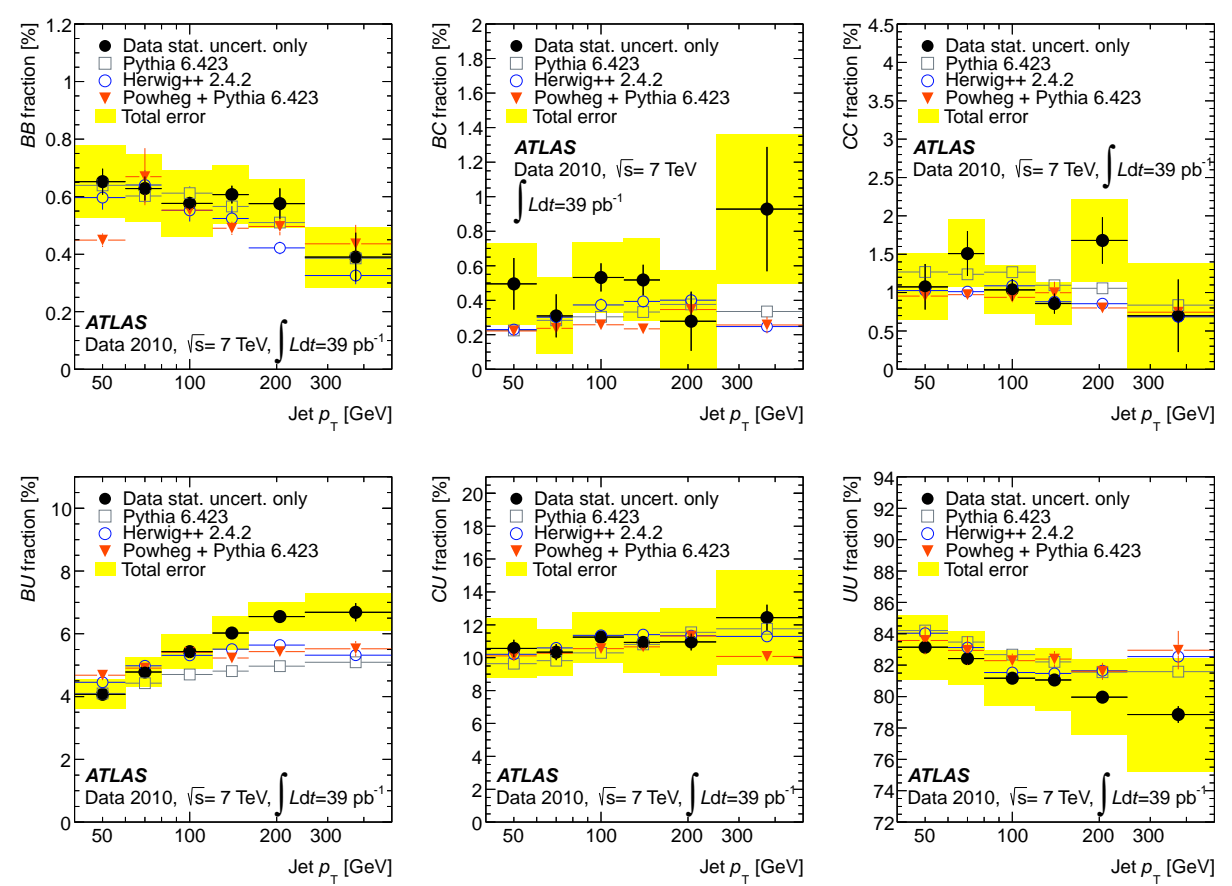

Figure 3: The measured dijet flavour composition for each leading jet $p_{\mathrm{T}}$ bin, compared with the MC predictions from PYTHIA, HERWIG++ and POWHEG+PYTHIA. [1]

\section{Conclusions}

An analysis of the jet flavour composition of dijet events has been performed and the composition of six flavour combinations of $B B, C C, U U, B U, C U$ and $B C$ is measured. Dijet events are analysed by a template fit in which kinematic information of secondary vertices in jets is utilised to separate light, charm and bottom jets. The results are compared to the predictions from NLO and LO MCs. The bottom jet asymmetry is well reproduced by the NLO MC prediction. Measured bottom-light flavour fraction is found to be larger than any of the predictions at jet $p_{\mathrm{T}}>100 \mathrm{GeV}$, while other flavour fractions are reproduced by the predictions.

\section{References}

[1] ATLAS Collaboration, Eur. Phys. J. C 73 (2013) 2301, arXiv:1210.0441.

[2] ATLAS Collaboration, JINST 3 (2008) S08003.

[3] T. Sjostrand, S. Mrenna and P. Z. Skands, JHEP 0605 (2006) 026, arXiv:hep-ph/0603175.

[4] A. Sherstnev and R. S. Thorne, Eur. Phys. J. C 55 (2008) 553, arXiv:0711.2473.

[5] ATLAS Collaboration, New J. Phys. 13 (2011) 053033, arXiv:1012.5104.

[6] ATLAS Collaboration, Eur. Phys. J. C 70 (2010) 823, arXiv:1005.4568.

[7] GEANT4 Collaboration, Nucl. Instrum. Meth. A 506 (2003) 250.

[8] M. Bahr et al., Eur. Phys. J. C 58 (2008) 639, arXiv:0803.0883.

[9] S. Alioli, K. Hamilton, P. Nason, C. Oleari and E. Re, JHEP 1104 (2011) 081, arXiv:1012.3380. 